\title{
Oscillation of Solutions to Fractional Partial Differential Equations with Several Delays
}

\author{
Yongfu Xiong, Li Xiao ${ }^{*}$ and Anping Liu \\ School of Mathematics and Physics, China University of Geosciences, Wuhan, Hubei, 430074, China \\ *Corresponding author
}

\begin{abstract}
In this paper, we study a class of nonlinear fractional partial differential equations with several delays to the second boundary condition. Based on properties of the RiemannLiouville fractional derivative, we establish a sufficient oscillatory condition of all solutions. The result is illustrated by an example.
\end{abstract} delay

Keywords-oscillation; fractional partial differential equation;

\section{INTRODUCTION}

Fractional differential equations are generalizations of classical differential equations of integer order. In the last few decades, fractional equations have gained considerable popularity and importance because of their applications in widespread fields of science and engineering, especially in mathematical modeling and simulation of system and processes. Nowadays, some aspects of fractional differential equations, such as the existence, the uniqueness and stability of solutions, the methods for explicit and numerical solutions have been investigated, we refer to [17-20].

In recent years, oscillatory behavior of solutions of fractional ordinary differential equations have been studied by authors [3-11]. However, there is a scarcity in the study of oscillation theory of fractional partial differential equations up to now, we refer to [12-16].

In this article, we are concerned with the oscillation of solutions to the fractional differential equations with several delays of the form

$$
\begin{gathered}
D_{+, t}^{1+\alpha} u(t, x)+p(t) D_{+, t}^{\alpha} u(t, x)=a(t) h(u) \Delta u \\
+\sum_{i=1}^{m} a_{i}(t) h_{i}\left(u\left(t-\tau_{i}(t), x\right)\right) \Delta u\left(t-\tau_{i}(t), x\right) \\
-\sum_{j=1}^{n} q_{j}(t, x) f_{j}\left(u\left(t-\delta_{j}(t), x\right)\right)+g(t, x),
\end{gathered}
$$$$
(t, x) \in R^{+} \times \Omega \equiv G
$$

with the boundary condition

$$
\partial u / \partial n=w(t, x, u),(t, x) \in R^{+} \times \partial \Omega .
$$

Where $\Omega$ is a bounded domain in $R^{n}$ with piecewise smooth boundary $\partial \Omega ; \alpha \in(0,1)$ is a constant; $G=R^{+} \times \Omega, R^{+}=(0,+\infty) \quad ; \quad D_{+, t}^{\alpha} u$ is the RiemannLiouville fractional derivative of order $\alpha$ of $u$ with respect of $t$; $\Delta$ is Laplacian operator; and $n$ is the unit exterior normal vector to $\partial \Omega$.

The following conditions are assumed to hold:

A. $\quad a(t), a_{i}(t), \tau_{i}(t), \delta_{j}(t) \in C\left(R^{+}, R^{+}\right)$ $p(t) \in C\left(R^{+}, R\right) ; 0<\tau_{i}(t)<\tau, 0<\delta_{i}(t)<\delta ; \delta, \tau$ are constants; $i=1,2 \mathrm{~L} m, j=1,2 \mathrm{~L} n$;

B. $q_{j}(t, x) \in C\left(\bar{G} ; R^{+}\right)$ and $q(t)=\min _{1 \leq j \leq n}\left(\min _{x \in \bar{\Omega}} q_{j}(t, x)\right)$;

C. $f_{j}: R \rightarrow R$ is a continuous function such that $f_{j}(u) / u \geq k_{j}>0$, for all $u \neq 0$, and $k_{j}$ is a positive constant;

D. $g \in C(\bar{G}, R)$;

E. $h(u), h_{i}(u) \in C(R, R) ; u h^{\prime}(u) \geq 0, u h_{i}^{\prime}(u) \geq 0$; $w(t, x, u)$ is a continuous function, such that $u w(t, x, u) h(u)<0, u w(t, x, u) h_{i}(u)<0$.

By a solution of the problem (1)-(2), we mean a function $u(t, x)$ which satisfies (1) on $\bar{G}$ and boundary condition (2).

A solution $u(t, x)$ of the problem (1)-(2) is said to be oscillatory in $G$ if it is neither eventually positive nor eventually negative, otherwise it is non-oscillatory. 


\section{PRELIMINARIES AND LEMMAS}

\section{A. Definition 1}

The Riemann-Liouville fractional partial derivative of order $\alpha>0$ with respect to $t$ of a function $u(t, x)$ is given by

$D_{+}^{\alpha} u(t, x):=\frac{1}{\Gamma(1-\alpha)} \frac{\partial}{\partial t} \int_{0}^{t}(t-v)^{-\alpha} u(v, x) d v, t>0$,

provided the right hand side is pointwise defined on $R^{+}$, Where $\Gamma$ is gamma function.

\section{B. Definition 2}

The Riemann-Liouville fractional integral of order $\alpha>0$ of a function $y: R^{+} \rightarrow R$ on the half-axis $R^{+}$is defined by

$$
\left(I_{+}^{\alpha} y\right)(t):=\frac{1}{\Gamma(\alpha)} \int_{0}^{t}(t-v)^{\alpha-1} y(v) d v, t>0
$$

provided the right hand side is pointwise defined on $R^{+}$.

\section{Definition 3}

The Riemann-Liouville fractional derivative of order $\alpha>0$ of a function $x$ on the half-axis $R^{+}$is defined by

$$
\begin{gathered}
D_{+}^{\alpha} x(t):=\frac{d^{\lceil\alpha\rceil}}{d t^{\lceil\alpha\rceil}} I_{+}^{\lceil\alpha\rceil-\alpha} x(t) \\
=\frac{1}{\Gamma(\lceil\alpha\rceil-\alpha)} \frac{d^{\lceil\alpha\rceil}}{d t^{\lceil\alpha\rceil}} \int_{0}^{t}(t-v)^{\lceil\alpha\rceil-\alpha-1} x(v) d v,
\end{gathered}
$$

$$
t>0
$$

provided the right hand side is pointwise defined on $R^{+}$. Where $\lceil\alpha\rceil$ is the ceiling function of $\alpha$.

D. Lemma 1

[2] Let $0<\alpha<1$ and $\left(I_{+}^{1-\alpha} y\right)(t)$ be the fractio-nal integral (4) of order $1-\alpha$, then

$$
\left(I_{+}^{\alpha} D_{+}^{\alpha} y\right)(t)=y(t)-\frac{\left(I_{+}^{1-\alpha} y\right)(0)}{\Gamma(\alpha)} t^{\alpha-1}
$$

[2]Let $0<\alpha<1, m \in N$ and $D=d / d x$. If the fractional derivatives $\left(D_{+}^{\alpha} y\right)(x)$ and $\left(D_{+}^{m+\alpha} y\right)(x)$ exist, then

$$
\left(D^{m} D_{+}^{\alpha} y\right)(x)=\left(D_{+}^{m+\alpha} y\right)(x)
$$

For the sake of convenience, in this article, we denote:

$$
\begin{gathered}
U_{1}(t)=\int_{\Omega} u(t, x) d x, \quad G_{1}(t)=\int_{\Omega} g(t, x) d x, \\
V(t)=\exp \int_{t_{0}}^{t} p(\xi) d \xi,
\end{gathered}
$$

\section{MAIN RESULT}

A. Theorem

Suppose that

$$
\lim _{t \rightarrow 0} I_{+}^{1-\alpha} U_{1}(t)=C_{1}
$$

where $C_{1}$ is a constant. If

$$
\liminf _{t \rightarrow \infty} \int_{0}^{t} \frac{(t-\xi)^{\alpha-1}}{V(\xi)}\left(C+\int_{t_{0}}^{\xi} G_{1}(s) V(s) d s\right) d \xi<0
$$

$$
\limsup _{t \rightarrow \infty} \int_{0}^{t} \frac{(t-\xi)^{\alpha-1}}{V(\xi)}\left(C+\int_{t_{0}}^{\xi} G_{1}(s) V(s) d s\right) d \xi>0
$$

then every solution of the problem (1)-(2) is oscillatory in $G$. Where $C$ is a constant.

Proof. Suppose to the contrary that there is a nonoscillatory solution $u(t, x)$ of the problem (1)-(2). Without loss of generality, we assume that there exists $T>0, t_{0}>T$, such that $u(t, x)>0$, for all $t \geq t_{0}$ and $u\left(t-\tau_{i}(t), x\right)>0$, $u\left(t-\delta_{j}(t), x\right)>0, i=1,2 \mathrm{~L} m, j=1,2 \mathrm{~L} n$.

Integrating (1) with respect to $x$ over the domain $\Omega$, we get

$$
\begin{gathered}
D\left(\int_{\Omega} D_{+, t}^{\alpha} u(t, x) d x\right)+p(t) \int_{\Omega} D_{+, t}^{\alpha} u(t, x) d x \\
=a(t) \int_{\Omega} h(u) \Delta u d x
\end{gathered}
$$

E. Lemma 2 
$+\sum_{i=1}^{m} a_{i}(t) \int_{\Omega} h(u)\left(t-\tau_{i}(t), x\right) \Delta u\left(t-\tau_{i}(t), x\right) d x$

$-\sum_{j=1}^{n} \int_{\Omega} q_{j}(t, x) f_{j}\left(u\left(t-\delta_{j}(t), x\right)\right) d x+\int_{\Omega} g(t, x) d x$,

$$
t>t_{0}
$$

Using Green's formula, boundary condition (2) and $E$ yield

$\int_{\Omega} h(u) \Delta u d x=\int_{\partial \Omega} h(u) \frac{\partial u}{\partial n} d s-\int_{\Omega} h^{\prime}(u)|\operatorname{gradu}|^{2} d x$

$=\int_{\partial \Omega} h(u) w(t, x, u) d s-\int_{\Omega} h^{\prime}(u)|\operatorname{gradu}|^{2} d x \leq 0$,

$$
\int_{\Omega} h_{i}\left(u\left(t-\tau_{i}(t), x\right)\right) \Delta u\left(t-\tau_{i}(t), x\right) d x \leq 0 .
$$

From $B$ and $C$, we can easily obtain

$$
\begin{aligned}
& \sum_{j=1}^{n} \int_{\Omega} q_{j}(t, x) f_{j}\left(u\left(t-\delta_{j}(t), x\right)\right) d x \\
& \geq \sum_{j=1}^{n} q(t) \int_{\Omega} f_{j}\left(u\left(t-\delta_{j}(t), x\right)\right) d x \\
& \geq \sum_{j=1}^{n} k_{j} q(t) \int_{\Omega} u\left(t-\delta_{j}(t), x\right) d x, \quad t \geq t_{0} .
\end{aligned}
$$

By Lemma 2.5, it follows from (12)-(15) that

$$
D_{+}^{1+\alpha} U_{1}(t)+p(t) D_{+}^{\alpha} U_{1}(t)
$$

$$
\leq-\sum_{j=1}^{n} k_{j} q_{j}(t) U_{1}\left(t-\delta_{j}(t)\right)+G_{1}(t)<G_{1}(t)
$$

$$
t \geq t_{0}
$$

According to (16) we can see that
$\left(\left(D_{+}^{\alpha} U_{1}(t)\right) V(t)\right)^{\prime}$

$$
=\left(D_{+}^{1+\alpha} U_{1}(t)\right) V(t)+p(t)\left(D_{+}^{\alpha} U_{1}(t)\right) V(t)
$$

$$
<G_{1}(t) V(t)
$$

$$
t \geq t_{0} \text {. }
$$

Integrating both sides of the above inequality from $t_{0}$ to $t$, we get

$$
\begin{aligned}
\left(D_{+}^{\alpha} U_{1}(t)\right) V(t) & <\left(D_{+}^{\alpha} U_{1}\left(t_{0}\right)\right) V\left(t_{0}\right)+\int_{t_{0}}^{t} G_{1}(s) V(s) d s \\
= & C+\int_{t_{0}}^{t} G_{1}(s) V(s) d s .
\end{aligned}
$$

where $C=\left(D_{+}^{\alpha} U\left(t_{0}\right)\right) V\left(t_{0}\right)$. From Lemma 2.4 and (18), we have

$$
\begin{gathered}
U_{1}(t)<\frac{I_{+}^{1-\alpha} U_{1}(0)}{\Gamma(\alpha)} t^{\alpha-1} \\
+I_{+}^{\alpha}\left(\frac{C}{V(t)}+\frac{1}{V(t)} \int_{t_{0}}^{t} G_{1}(s) V(s) d s\right) \\
=\frac{C_{1}}{\Gamma(\alpha)} t^{\alpha-1}
\end{gathered}
$$

$$
+\frac{1}{\Gamma(\alpha)} \int_{0}^{t} \frac{(t-\xi)^{\alpha-1}}{V(\xi)}\left(C+\int_{t_{0}}^{\xi} G_{1}(s) V(s) d s\right) d \xi
$$

Taking $t \rightarrow \infty$, from (19) and (10) we can obtain

$$
\liminf _{t \rightarrow \infty} U_{1}(t) \leq \limsup _{t \rightarrow \infty} \frac{C_{1}}{\Gamma(\alpha)} t^{\alpha-1}+\liminf _{t \rightarrow \infty} \frac{1}{\Gamma(\alpha)}
$$

$$
\cdot \int_{0}^{t} \frac{(t-\xi)^{\alpha-1}}{V(\xi)}\left(C+\int_{t_{0}}^{\xi} G_{1}(s) V(s) d s\right) d \xi<0 .
$$


which contradicts $U_{1}(t, x)>0$.

On the other hand, we assume that there exists $T>0$, $t_{0}>T$, such that $u(t, x)<0$ for all $t \geq t_{0}$, and $u\left(t-\tau_{i}(t), x\right)<0, u\left(t-\delta_{j}(t), x\right)<0, \quad i=1,2 \mathrm{~L} m$, $j=1,2 \mathrm{~L} n$. We also have (12). Using the similar methods, we can easily obtain

$$
\begin{gathered}
U_{1}(t)>\frac{I_{+}^{1-\alpha} U_{1}(0)}{\Gamma(\alpha)} t^{\alpha-1} \\
+I_{+}^{\alpha}\left(\frac{C}{V(t)}+\frac{1}{V(t)} \int_{t_{0}}^{t} G_{1}(s) V(s) d s\right) \\
=\frac{C_{1}}{\Gamma(\alpha)} t^{\alpha-1} \\
+\frac{1}{\Gamma(\alpha)} \int_{0}^{t} \frac{(t-\xi)^{\alpha-1}}{V(\xi)}\left(C+\int_{t_{0}}^{\xi} G_{1}(s) V(s) d s\right) d \xi
\end{gathered}
$$

Taking $t \rightarrow \infty$, from (21) and (11) we can obtain

$$
\begin{gathered}
\limsup _{t \rightarrow \infty} U_{1}(t) \geq \liminf _{t \rightarrow \infty} \frac{C_{1}}{\Gamma(\alpha)} t^{\alpha-1}+\limsup _{t \rightarrow \infty} \frac{1}{\Gamma(\alpha)} \\
\quad \cdot \int_{0}^{t} \frac{(t-\xi)^{\alpha-1}}{V(\xi)}\left(C+\int_{t_{0}}^{\xi} G_{1}(s) V(s) d s\right) d \xi>0 .
\end{gathered}
$$

Which contradicts $U_{1}(t, x)<0$. The proof is completed.

\section{Example}

Consider the fractional differential equation

$$
\begin{gathered}
\frac{\partial}{\partial t}\left(D_{+, t}^{1 / 2} u(t, x)\right)-D_{+, t}^{1 / 2} u(t, x) \\
=u^{2}(t, x) \Delta u(t, x)+u^{2}\left(t-\frac{\pi}{2}, x\right) \Delta u\left(t-\frac{\pi}{2}, x\right)
\end{gathered}
$$

$$
\begin{aligned}
\left.-u\left(t-\frac{\pi}{2}, x\right)-\left(x^{2}+t^{2}+1\right) u\left(t-\frac{2 \pi}{3}, x\right) e^{\left[u\left(t-\frac{2 \pi}{3}, x\right)\right.}\right]^{2} \\
+\frac{1}{2} e^{t} \sin t \sin x, \quad(t, x) \in R^{+} \times(0, \pi),
\end{aligned}
$$

with the boundary condition

$$
\frac{\partial u(t, x)}{\partial n}=-u(t, x), \quad x=0, \pi, t \in R^{+} .
$$

where $\alpha=1 / 2, \Omega=(0, \pi), n=1, p(t)=-1$, $a(t)=a_{i}(t)=1, \quad h(u)=h_{i}(u)=u^{2}, \quad q_{1}(t, x)=1$, $q_{2}(t, x)=t^{2}+x^{2}+1 \quad, \quad f_{1}(u)=u \quad, \quad f_{2}(u)=u e^{u^{2}}$, $g(t, x)=\frac{1}{2} e^{t} \sin t \sin x, w(t, x, u)=-u$.

It is easy to verify that the conditions $A-E$ are satisfied, and $V(t)=e^{t_{0}-t}$, and

$$
G_{1}(t)=\int_{\Omega} f(t, x) d x=\int_{0}^{\pi} e^{t} \sin t \sin x d x=e^{t} \sin t
$$

Hence

$$
\begin{array}{r}
\int_{t_{0}}^{\xi} G_{1}(s) V(s) d s=\int_{t_{0}}^{\xi}\left(e^{s} \operatorname{sins}\right) e^{t_{0}-s} d s \\
=e^{t_{0}}\left(-\cos \xi+\cos t_{0}\right) .
\end{array}
$$

Let $t_{0}=\pi / 2$, we have

$$
\int_{0}^{t} \frac{(t-\xi)^{-1 / 2}}{V(\xi)}\left(C+\int_{t_{0}}^{\xi} G_{1}(s) V(s) d s\right) d \xi
$$

$$
=\int_{0}^{t}(t-\xi)^{-1 / 2} e^{\xi-\pi / 2}\left(C-e^{\pi / 2} \cos \xi\right) d \xi .
$$

It is easy to verify (10) and (11) hold. Hence all solutions of the problem (23)-(24) oscillate.

\section{ACKNOWLEDGMENT}

The authors thanks the referees very much for their valuable comment and suggestions on this paper. 


\section{REFERENCES}

[1] K. S. Miller, B. Ross, "An introduction to the fractional calculus and fractional differential equations,” John Wiley Sons, New York, 1993.

[2] A. A. Kilbas, H. M. Srivastava, J. J. Trujillo, "Theory and applications of fractional differential equations,” Elsevier Science BV, Amsterdam, 2006.

[3] D. X. Chen, "Oscillation criteria of fractional differential equations" Advances in Difference Equations, vol. 33, pp. 1-18, 2012.

[4] Z. Han, Y. Zhao, Y. Sun and C. Zhang, "Oscillation for a class of fractional differential equation," Discrete Dynamics in Nature and Society, vol. 2013, Article ID 390282, 6 pages, 2013.

[5] D. X. Chen, "Oscillatory behavior of a class of fractional differential equations with damping,” UPB Scientific Bulletin Series A, vol. 75, pp. 107-118, 2013.

[6] S. R. Grace, R. P. Agarwal, "On the oscillation of fractional differential equations,” Fractional Calculus and Applied Analysis, vol. 15, pp. 222231, 2012.

[7] J. C. Yang, A. P. Liu, and T. Liu, "Forced oscillation of nonlinear fractional differential equations with damping term," Advances in Difference Equations, vol. 2015, 7 pages, 2015.

[8] B. Zheng, "Oscillation for a class of nonlinear fractional differential equations with damping term,” Journal of Advanced Mathematical Studies, vol. 6, pp. 107-115, 2013.

[9] Q. H. Feng, "Inerval oscillation criteria for a class of nonlinear fractional differential equations with nonlinear damping term," International Journal of Applied Mathematics, vol. 43, pp. 3-9, 2013

[10] D. X. Chen, P. X. Qu, and Y. H. Lan, "Forced oscillation of certain fractional differential equations,” Advances in Difference Equations, vol 2013, article 125, 10 pages, 2013.

[11] Q. H. Feng, F. W. Meng, "Oscillation of solutions to nonlinear forced fractional differential equations," Electronic Journal of Differential Equations, vol. 2013, pp. 1-10, 2013.

[12] S. Harikrishnan, P. Prakash, and J. J.Nieto, "Forced oscillation of solutions of a nonlinear fractional partial differential equation,” Applied Mathematics and Computation, vol. 254, pp. 14-19, 2015.

[13] P. Prakash, S. Harikrishnan, J. J. Nieto, and J.-H. Kim, “Oscillation of a time fractional partial differential equation," Electronic Journal of Qualitative Theory of Differential Equations, vol. 15, pp. 1-10, 2014.

[14] P. Prakash, S. Harikrishnan, and M. Benchohra, "Oscillation of certain nonlinear fractional partial differential equation with damping term," Applied Mathematics Letters, vol. 43, pp. 72-79, 2015.

[15] W. N. Li, "Forced oscillation criteria for a class of fractional partial differential equations with damping term," Mathematical Problems in Engineering, vol. 2015, Article ID 410904, 6 pages, 2015.

[16] W. N. Li, "On the forced oscillation of certain fractional paritial differential equations,” Applied Mathematics Letters, vol. 50, pp. 5-9, 2015.

[17] M. Benchohra, J. Henderson, S. K. Ntoyuas, A. Ouahab, "Existence results for fractional order functional differential euqations with infinite delay,” Journal of Mathematical Analysis and Applications, vol. 338, pp. 1340-1350, 2008.

[18] D. Delbosco, L. Rodino, "Existence and uniqueness for a nonlinear fracti0nal differential equatin,” Journal of Mathematical Analysis and Applications, vol. 204, pp. 609-625, 1996.

[19] W. Deng, "Smootheness and stability of the solutions for nonlinear fractional differential equations," Nonlinear Analysis, vol. 72, pp. 17681777, 2010.

[20] L. Galecoe, R. Garrappa, "Explicit methods for fractional differential equations and their proerties," Joural of Computational and Applied Mathematics, vol. 228, pp. 548-560, 2009.

[21] A. P. Liu, M. X. He, "Osicllatory properties of the solutions of nonlinear delay hyperbplic differential equations of neutral type," Applied Mathematics and MEchanics, vol. 23, pp. 678-685, 2002. 